\title{
Spotlight on anifrolumab and its potential for the treatment of moderate-to-severe systemic lupus erythematosus: evidence to date
}

This article was published in the following Dove Press journal:

Drug Design, Development and Therapy

\author{
Renaud Felten ${ }^{1-3}$ \\ Florence Scher ${ }^{4}$ \\ Flora Sagez ${ }^{1,2}$ \\ François Chasset ${ }^{5}$ \\ Laurent Arnaud 1,2,6
}

\begin{abstract}
'Rheumatology Department, University Hospital of Strasbourg, Université de Strasbourg, Strasbourg, F-67000, France; ${ }^{2}$ National Reference Centre for Rare Systemic and Autoimmune Diseases East South-West (RESO), Strasbourg, France;

${ }^{3}$ Immunology Laboratory,

"Immunopathologie et Chimie

Thérapeutique", Institut de Biologie Moléculaire et Cellulaire (IBMC), CNRS UPR3572, Strasbourg, F-67000, France; ${ }^{4}$ Pharmacy-Sterilisation Department, University Hospital of Strasbourg. University of Strasbourg, Strasbourg, France; ${ }^{5}$ Faculty of Medicine at Sorbonne University, AP-HP, Dermatology and Allergology Department, Tenon Hospital, Sorbonne University, Paris, F-75020, France; ${ }^{6}$ Immuno-Rheumatology Laboratory, "Laboratoire d'ImmunoRhumatologie Moléculaire", INSERM UMR_SI 109, Strasbourg, F-67000, France
\end{abstract}

Abstract: Previous reports have described the appearance of systemic lupus erythematosus (SLE) cases following interferon- $\alpha(\mathrm{IFN}-\alpha)$ therapy, IFN-regulated gene expression is significantly increased in SLE, and an association between SLE and gene variants belonging to IFN downstream pathways has been shown. Based on this, targeting of IFN and of their signaling pathways has appeared to be interesting developments within the field of SLE therapy. Different specific type I IFN antagonists have been studied in clinical trials and some of those have already reached Phase III. A potential approach would be to target IFN receptors rather than IFN themselves. Anifrolumab (previously MEDI-546) is a fully human monoclonal antibody $(\mathrm{Ab})$ that binds to subunit 1 of the type I IFN receptor (IFNAR1), blocking the action of different type I IFNs (IFN- $\alpha$, IFN- $\beta$ and IFN- $\omega$ ). This drug has been assessed in 11 clinical studies: 9 in SLE, 1 in systemic sclerosis and 1 in rheumatoid arthritis. In SLE, clinical development reached Phase I for 1 study and Phases II and III for 5 and 3 trials, respectively. The Phase IIb, randomized control trial (RCT), double-blind, placebo-controlled study of adults with moderate-to-severe SLE (MUSE trial) showed positive results on the composite primary endpoint SRI-4. Greater efficacy was seen in patients with high baseline IFN gene signature compared with those with low baseline IFN gene signature. Anifrolumab also demonstrated promising results on cutaneous and arthritic manifestations, especially among patients with a high IFN gene signature. The pivotal Treatment of Uncontrolled Lupus via the Interferon IFN Pathway (TULIP 1 and 2 studies are now completed. In August 2018, the promoter announced that the TULIP 1 Phase III trial did not reach its primary endpoint. The release of the completed but not yet published Phase II studies and of the TULIP pivotal trials results will further inform us on the actual therapeutic potential of anifrolumab.

Keywords: systemic lupus erythematosus, interferon type I, interferon-alpha, anifrolumab, receptors interferon

\section{Introduction}

The last decades have been marked by a profound improvement in the prognosis of patients with systemic lupus erythematosus (SLE), thanks to changes in the pharmacopeia. ${ }^{1}$ The survival is currently higher than $90 \%$ at 10 years in most dedicated centers. For 60 years, the only new treatment approved in the SLE has been belimumab, despite 74 targeted therapies being studied in SLE. ${ }^{2}$ In parallel, new pathways involved in the physiopathology of SLE have emerged, leading to the recognition of interferons (IFNs) as critical mediators in SLE. Therefore, new therapeutic strategies targeting IFNs have been developed in SLE. ${ }^{3}$
Correspondence: Laurent Arnaud Service de Rhumatologie, Centre National de Référence des Maladies Autoimmunes et Systémiques Rares, Hôpital de Hautepierre, I Avenue Molière BP 83049, Strasbourg Cedex 67098, France

Tel +3338 812 8474

$\mathrm{Fax}+33388128290$

Email laurent.arnaud@chru-strasbourg.fr 


\section{From IFNs to IFN-blocking strategies}

IFNs have been discovered as antiviral cytokines in $1957 .{ }^{4}$ About 20 subtypes have now been described and divided into three main families: type I, type II and type III. Type I IFNs represent the largest family, with IFN- $\alpha$ and IFN- $\beta$ being involved in antiviral immunity but also in inflammatory pathways. ${ }^{5}$

Several experiments of mouse models and SLE patients have highlighted the role of type I IFN and particularly IFN$\alpha$ in SLE. Stimulation of the production of type I IFN in NZB/NZWF1 mice, one of the most interesting mouse models of SLE, by polyinosinic:polycytidylic acid, induces production of antinucleic acid antibodies and tissue damage. ${ }^{6}$ Subsequently, the early observation of increased type I IFN levels in SLE patients was described. ${ }^{7}$ Likewise, patients with carcinoid tumors treated with IFN therapy showed an increased incidence of autoimmune diseases such as SLE. ${ }^{8}$ At the beginning of the 2000s, large gene transcripts induced by type I IFN has been characterized from the blood of SLE patients. ${ }^{9,10}$ Genome-wide association studies (GWASs) of SLE patients have thereafter identified the important role of gene variants encoding for proteins of the innate immune response, such as TLR-7, TLR-9, IRF-5, IRF-7 and IRF-8. ${ }^{11}$ In the recent past, it has been shown that Aicardi-Goutières syndrome, a disease related to the upregulation of type I IFN, shared several SLE features, leading to the identification of monogenic forms of SLE. ${ }^{12}$ As a consequence, a group of different Mendelian diseases characterized by an upward regulation of type I IFN has emerged in 2011 and was termed "interferonopathies". ${ }^{13}$

IFN-I-induced gene transcripts signature is now extensively known as the "interferon signature". Genome-wide gene expression studies using microarrays led to an identification of a dysregulated expression of genes in the IFN pathway in about $50-75 \%$ of SLE patients. ${ }^{9,14}$ Type I IFN signaling pathway was found to be the most highly activated signaling pathway in whole blood of both pediatric and adult SLE patients. ${ }^{15,16}$ More recently, different sets of genes derived from genome-wide gene expression studies and validated by quantitative PCR were developed to measure IFN gene expression based on a limited number of genes. ${ }^{9,14-18}$ These sets of genes were used as quantitative $e^{9,15,18}$ or qualitative scores (eg, high versus low IFN gene signature). ${ }^{14-17}$ A high IFN gene signature was found to be more frequently in patients with severe phenotypes such as involvement of the central nervous system, hematological disorders and/or renal disease. ${ }^{9}$ Moreover, both quantitative and qualitative
IFN scores were found to be associated with the Systemic Lupus Erythematosus Disease Activity Index (SLEDAI) score. ${ }^{16-18}$ However, the potential role of a high IFN gene signature to predict patients at risk of flare remains controversial. Indeed, a high IFN gene signature was shown to be an independent predictor of flare over 52 weeks in the Phase III trials of tabalumab (an anti-BLyS monoclonal antibody [Ab]) involving 1,760 patients; ${ }^{14}$ however, this was not confirmed in a longitudinal study assessing changes in IFN gene signature with focus on flare events. ${ }^{18}$

On the grounds of data from mouse SLE models, the occurrence of SLE following IFN therapy, IFN signature and the recognition of monogenic SLE associated with an upward regulation of type I IFN has underlined the opportunity to develop novel drugs targeting IFN and their downstream pathways in $\mathrm{SLE}^{3}$ (Figure 1). In light of these observations, specific type I IFN antagonists have been studied in Phase III clinical trials. ${ }^{3}$ In a second step, other approaches, such as targeting of downstream IFN- $\alpha$ pathways or the blockade of IFN- $\gamma$, are being studied in SLE, at an earlier development stage. ${ }^{3}$

Type I IFN proteins bind to the subunit 1 of type I IFN heterodimeric transmembrane receptor (IFNAR1). IFNAR is composed of the two subunits IFNAR1 and IFNAR2 that will activate Janus kinase 1 (JAK1) and tyrosine kinase 2 (TYK2) (Figure 1). These kinases lead to the phosphorylation of IFNAR, resulting in the involvement of signal transducer and activator of transcription (STAT) proteins and then phosphorylation, dimerization and nuclear translocation of STAT. In the nucleus, STAT is a transcription factor which induces the expression of IFN-regulated genes (IRGs). ${ }^{19}$ In this context, JAK inhibitors appear as possibly worthwhile SLE medications. Several molecules are actually under clinical evaluation such as baricitinib (completed Phase II), BMS986165 (anti-TYK2, recruiting Phase II), filgotinib (recruiting Phase II), solcitinib (terminated Phase II) and tofacitinib (recruiting Phase I/II) (Figure 1). In its Phase II study, baricitinib showed interesting positive results with greater symptoms improvement than placebo in patients with active SLE as an add-on of standard of care (SOC) therapy. ${ }^{20}$

\section{Structure and pharmacokinetic (PK) of anifrolumab}

Anifrolumab (previously MEDI-546), a fully human monoclonal $\mathrm{Ab}$, blocks the action of different type I IFNs such as IFN- $\alpha$, IFN- $\beta$ and IFN- $\omega^{21}$ by binding to the subunit 1 of the type I interferon receptor (IFNAR1). IFNAR1 is a helical 


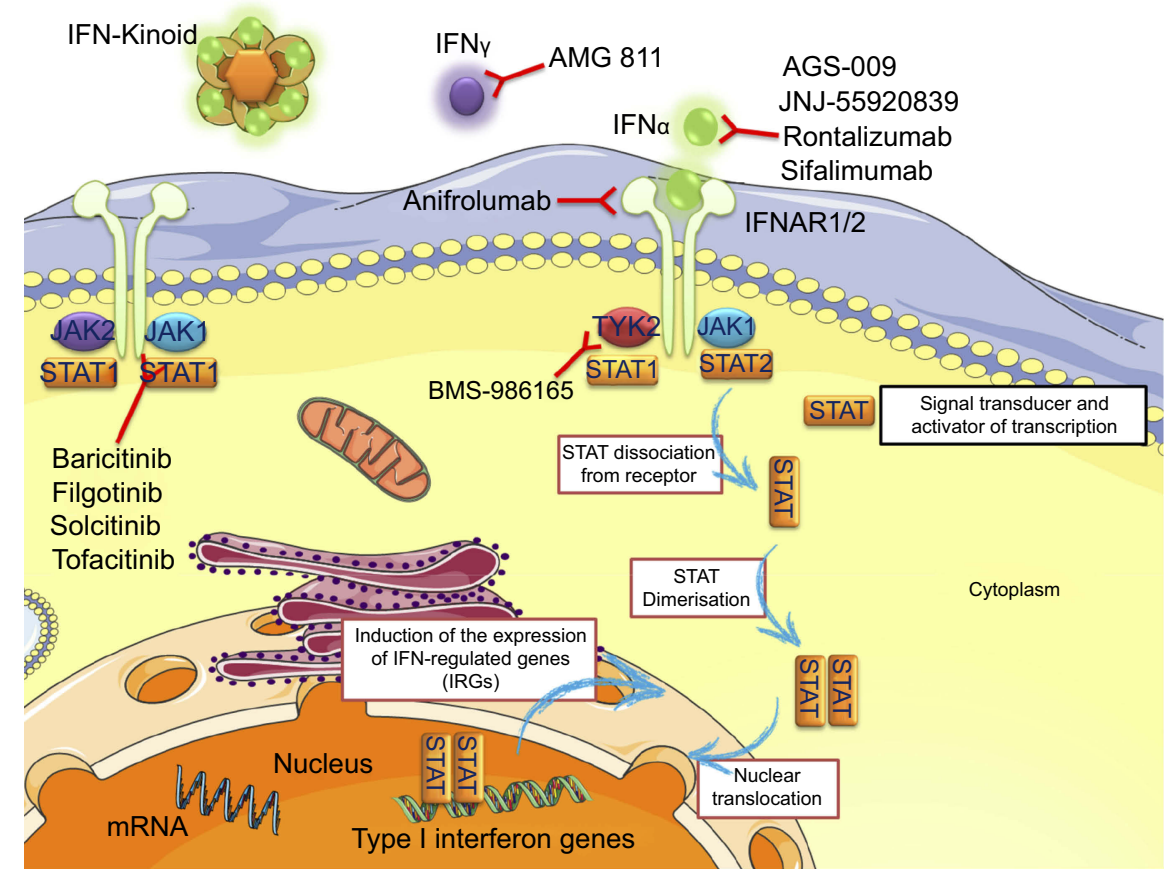

Figure I Drugs targeting of IFNs and their downstream pathways in SLE.

Abbreviations: IFN, Interferon; IFNAR, Interferon-alpha/beta receptor; JAK, Janus Kinase; mRNA, messenger ribonucleic acid; STAT, signal transducers and activators of transcription; TYK2, Tyrosine Kinase 2.

cytokine receptor composed of 4 fibronectin type III subdomains divided into 2 domains (1: SD1+SD2 and 2: SD3+SD4), an intracellular domain and a single-span transmembrane domain. $^{22,23}$ A ternary signaling complex is formed by IFNAR1, IFNAR2 and IFN that leads to the activation of signal transduction pathways. Anifrolumab has a constant domain (Fc domain) composed with 3 modifications L234F, L235E and P331S in order to reduce Ab Fc-mediated agonist actions. $^{24,25}$ The epitope of anifrolumab is located on a $12-\mathrm{kDa}$ fragment at the SD3+SD4 IFNAR1 subdomains with $\mathrm{N}$-terminal residue $\mathrm{R}$, and more precisely in the SD3 subdomain (amino acids $\mathrm{Y}^{276} \mathrm{~L}^{277} \mathrm{R}^{279}$ ). ${ }^{26}$ Others report that IFNAR1-antagonist Ab binds to the subunit 1 of IFNAR1. ${ }^{26-28}$

Anifrolumab induces rapid internalization of IFNAR1 from the surface of monocytes and thereby reduces the occurrence of heterodimerization with IFNAR2 and the setting up of the IFN signaling complex. By antagonizing the binding of the different IFNs to the IFNAR1 receptor, anifrolumab blocks STAT1 phosphorylation and IFN-stimulated response element (ISRE) activity. Dose-dependently, anifrolumab inhibits type I IFN production and the production in a lesser extent of some cytokines (TNF- $\alpha$, IL- 6 and IL- 8 by 40-50\%) and reduces CD80 and CD83 expression of dendritic cells by $30-50 \% .^{29}$ Blockade of IFNAR1 also alters the type I IFN autoamplification, mediated by the cell- intrinsic induction related to IFNAR activation. ${ }^{30,31}$ Anifrolumab also inhibits the differentiation of B-cells into plasma cells by interfering with the production of IFN. ${ }^{29}$

Anifrolumab administered at $300 \mathrm{mg}$ intravenously every 4 weeks for 48 weeks is able to neutralize $85-90 \%$ of the IFN signature (median range) from 29 to 365 days. $^{32}$ Maximum concentration (Cmax) and exposure (AUC) of $300 \mathrm{mg}$ and $600 \mathrm{mg} \mathrm{SC}$ anifrolumab change dose-proportionally. Peak serum concentrations are reached 4-7 days after injection. The exposure of $300 \mathrm{mg}$ SC anifrolumab is about $87 \%$ of the intravenous (IV) one. Its concentrations are detectable for approximately 1 month postadministration and below the limit of detection by 84 days postdose. ${ }^{33}$

Anifrolumab has been or is currently being studied in 11 different studies (Table 1): 9 in SLE, 1 in systemic sclerosis (NCT00930683) and 1 in rheumatoid arthritis (NCT03435601). In SLE, clinical development reached Phase I in 1 study, Phase II in 5 and Phase III in 3 studies.

\section{Clinical studies with anifrolumab Phase I clinical study (NCT02601625 ${ }^{34}$ )}

Anifrolumab has been assessed in a Phase I, single-center, double-blind, randomized control trial (RCT) versus placebo 


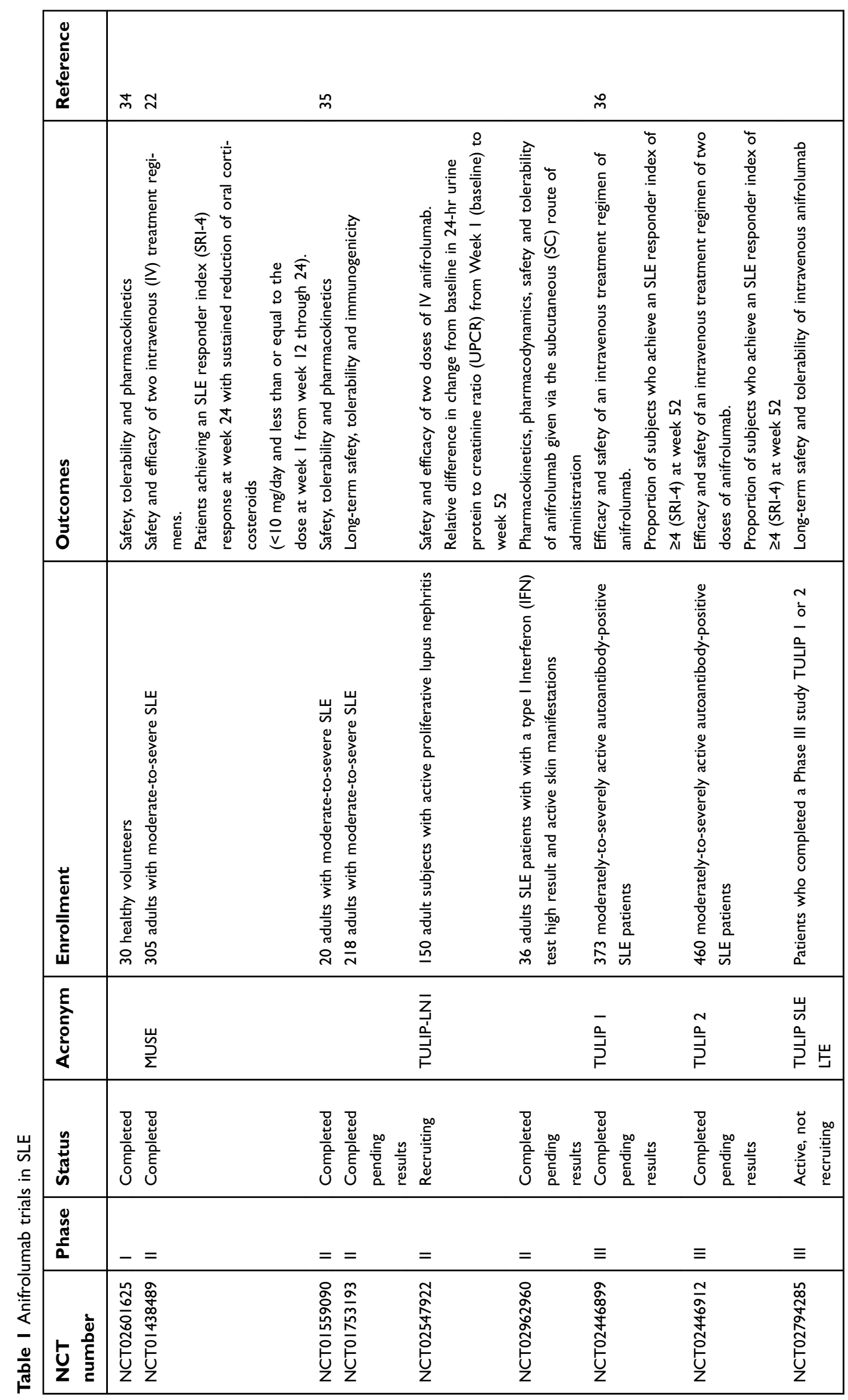


trial (NCT02601625) involving healthy volunteers. ${ }^{33}$ Thirty healthy adults were randomized and 28 (93\%) completed the study. The aim of this trial was to establish the safety, PK, tolerability and immunogenicity of subcutaneous (SC) or IV doses of anifrolumab. SC injection of $300 \mathrm{mg}$ and $600 \mathrm{mg}$ demonstrated dose-proportional PK. Both SC and IV routes of administration were well tolerated. Adverse events (AEs) were present in, respectively, $50 \%(n=9)$ and $33 \%(n=4)$ of patients in anifrolumab and placebo groups. The most frequent $\mathrm{AE}$ in the anifrolumab group was upper respiratory tract infection $(n=3 ; 17 \%)$ and dry throat $(n=2 ; 11 \%)$. Antidrug $\mathrm{Ab}$ was detected in 1 patient of the IV administration group (300 mg) at Day 84. No serious AEs were noticed.

\section{Phase II clinical studies (NCTOI43848922, NCTOI 559090, ${ }^{35}$ NCTOI753193, NCT02547922 and NCT02962960)}

Following this $\mathrm{PK}$, tolerance and safety study, anifrolumab was then studied in a Phase IIb, multicenter, double-blind, placebocontrolled RCT (MUSE trial [NCT01438489] ${ }^{22}$ ). This trial included adults with moderate-to-severe SLE but, of note, neuropsychiatric SLE and lupus nephritis were exclusion criteria. Three hundred and five patients were randomized between IV dose of anifrolumab (300 mg or $1 \mathrm{~g}$ ) and placebo. Doses were administrated every month for 48 weeks, as an add-on-therapy to SOC. A stratified randomization was applied according to oral glucocorticoid dosage $(\geq 10$ or $<10 \mathrm{mg} /$ day $)$, SLEDAI-2K $(\geq 10$ or $<10)$ and type I IFN gene signature results at baseline. The primary endpoint was the percentage of patient, both reaching a sustained reduction of oral corticosteroids and an SLE responder index (SRI-4) response at week 24 . Notably, this primary endpoint was investigated in a modified intent-to-treat population as well as in the type I IFN-high subpopulation (2-sided type I error rate set to 0.10 ). The trial was considered positive if the primary endpoint was fulfilled in either of these 2 situations. At last, the percentage of patients who fulfilled the primary endpoint was higher in the anifrolumab group (34.3\% for $300 \mathrm{mg}, p=0.014$ and $28.8 \%$ for $1 \mathrm{~g}, p=0.06$ ) than the placebo group (17.6\%). There was no significant difference between the groups in terms of global tolerance and safety despite a more frequent Herpes zoster reactivation $(5.1 \%$ with $300 \mathrm{mg}$ and $9.5 \%$ with $1 \mathrm{~g}$ versus $2.0 \%$ with placebo), and also more influenza in anifrolumab-treated patients.

Other Phase II studies include:

NCT01559090, a Phase II, multicenter, open-label, dose-escalation study to evaluate the safety and tolerability of IV dose of anifrolumab in Japanese subjects with active SLE, has been completed. ${ }^{35}$ The primary outcome was the description of the safety profile of anifrolumab at dosages up to $1,000 \mathrm{mg}$ IV every 4 weeks for 48 weeks and $300 \mathrm{mg}$ IV every 4 weeks for 156 weeks, among Japanese patients. Twenty patients were enrolled and 17 received treatment. IV anifrolumab was well tolerated with a decent safety profile at the dose of either 100,300 or $1,000 \mathrm{mg}$. Noteworthy, a pulmonary embolism occurred 9 weeks after anifrolumab was stopped, and a cerebral infarction occurred in a patient with a history of previous cerebral vasculitis and two events of cerebral infarction. An obvious assessment of causality was not figured out for these two thrombotic events occurred during the study. There were no statistical trends to suggest dose-dependent safety. Throughout the study period, all patients suffered at least one $\mathrm{AE}$, most of moderate intensity (grade 1 or $2)$. The most common AEs were nasopharyngitis $(\mathrm{n}=8$, $47.1 \%)$, upper abdominal pain $(n=4,23.1 \%)$ and headache $(n=3,17.6 \%) .{ }^{35}$ SLE disease activity seemed to improve after anifrolumab therapy. Nevertheless, in this noncontrolled versus placebo trial, it is difficult to draw efficacy conclusions.

NCT01753193, an open-label extension study to evaluate long-term safety and tolerability of IV anifrolumab in adult subjects with moderately-to-severely active SLE, has also been completed, with pending results.

Anifrolumab is also being tested in a Phase II trial for lupus nephritis and a Phase II trial using SC delivery in SLE.

NCT02547922, TULIP-LN1, a multicenter, doubleblind, placebo-controlled, RCT, Phase II study assessing the efficacy and safety of anifrolumab in active proliferative lupus nephritis, is currently ongoing (recruiting).

NCT02962960, a multicenter, randomized, doubleblind, placebo-controlled, Phase II study characterizing the PK, pharmacodynamics (PD) and safety of anifrolumab following SC administration in adult SLE subjects with type I IFN test high result and active skin manifestations, is currently ongoing (active, not recruiting).

\section{Phase III clinical studies (NCT02446899, NCT024469I 2 and NCT02794285)}

The pivotal TULIP (Treatment of Uncontrolled Lupus via the IFN Pathway) programme contains two Phase III clinical trials, TULIP 1 and TULIP 2, which were evaluating the efficacy and safety of anifrolumab versus placebo in 
moderate-to-severe active autoAb-positive SLE patients receiving SOC treatment.

TULIP 1 (NCT02446899), a Phase III, multicenter, multinational, double-blind, placebo-controlled RCT, randomized 460 patients $(1: 2: 2)$ to receive a fixed-dose IV infusion of $150 \mathrm{mg}$ anifrolumab, $300 \mathrm{mg}$ anifrolumab or placebo every 4 weeks.

TULIP 2 (NCT02446912), a Phase III, multicenter, multinational, double-blind, placebo-controlled RCT, randomized 373 patients $(1: 1)$ to receive a fixed-dose IV infusion of $300 \mathrm{mg}$ anifrolumab or placebo every 4 weeks.

These trials evaluate the effect of anifrolumab in lowering disease activity (assessed by the SRI-4) and the use of oral corticosteroids, improving skin manifestations (assessed by cutaneous lupus erythematosus disease area and severity index (CLASI), and reducing flares.

The SRI-4 is a composite endpoint, defined by the following criteria:

- Reduction from baseline of $\geq 4$ points in the SLEDAI$2 \mathrm{~K}$;

- No new organ system affected as defined by 1 or more British Isles Lupus Assessment Group (BILAG) 2004 A or 2 or more BILAG-2004 B items compared to baseline using BILAG-2004;

- No worsening from baseline in subjects' lupus disease activity defined by an increase $\geq 0.30$ points on a 3-point physician's global assessment VAS and

- No discontinuation of investigational product or use of restricted medications beyond the protocol allowed threshold before assessment.

Importantly, the promoter announced on 31 August that the TULIP 1 Phase III trial did not meet the primary endpoint of a statistically significant reduction in disease activity in patients with SLE as measured by the SRI-4 at 12 months, but the detailed results are still pending. ${ }^{36}$

In addition to the pivotal trials, anifrolumab is also being tested in a Phase III SLE long-term extension trial, TULIP SLE LTE (NCT02794285), a multicenter, multinational, double-blind, placebo-controlled extension study to characterize the long-term safety and tolerability of an IV anifrolumab-therapy versus placebo as an add-on to SOC, in moderate-to-severe active SLE who completed a TULIP 1 or 2 study through the 52-week double-blind treatment period.

\section{Discussion}

Previous studies have described the appearance of SLE cases following IFN- $\alpha$-therapy, and IRGs expression is significantly increased in SLE and an association between SLE and gene variants belonging to IFN downstream pathways has been shown. Based on this, targeting of IFNs and of their signaling pathways has appeared to be interesting developments within the field of SLE therapy.

One of the important contemporary challenges in the management of SLE is developing more effective drugs on the grounds of the lack of efficient targeted therapies to treat the heterogeneous SLE manifestations. For 60 years, the only new treatment approved in the SLE has been belimumab. Crucial evaluations from investigators involved in RCTs have made it possible to emphasize the several obstacles to effective drug development in SLE. These barriers could be represented by SLE manifestations heterogeneity, incomplete PK/PD and dose evaluation before onset of broad RCTs, the addition of evaluated targeted therapies as an add-on-therapy to the SOC, the difficulties in managing background treatment in RCTs, inappropriate study design (in terms of size or duration) and probably also improper selection of primary endpoint. ${ }^{37}$ Several other treatments with positive results in Phase II trials have recently failed to fulfill primary endpoint in SLE Phase III RCTs. Nevertheless, learning from these unsuccessful RCTs allowed improvements in RCT SLE design. On the grounds of those obstacles to efficient drug development from the Phase II RCTs, pivotal studies of belimumab ${ }^{38,39}$ included only anti-DNA seropositive SLE patients and used the SRI-4 as a new primary endpoint. Even though this approach of composite responder indices does not appear to be perfect, it also has uncomfortable limitations in the context of RCTs. Much labor of the scientific community to work on the development of more efficient endpoints for SLE RCTs might allow capturing clinically relevant treatment responses. Touma and Gladman have recently discussed the most common pitfalls in the selection of patients and endpoints. ${ }^{40}$ Of note, the heterogeneity of lupus patients requires careful selection at inclusion to evaluate the therapeutic response of those with active lupus disease instead of the stigma of previous flares. This also implies the participation of expert centers used to assessing complex lupus patients and the training of investigators in the use of multiple disease activity instruments. As far as endpoints are concerned, reproducible and reliable criteria have to be 
used. Organ-specific instruments have to be encouraged whenever possible (CLASI for example). Finally, ongoing clinical trials are evaluating treatment as an add-on of SOC. However, the use of glucocorticoids can increase the efficacy in the placebo arm. Therefore, the use and dosage of glucocorticoids should be limited and monitored.

An important point is that the score used to assess IFN gene signature in anifrolumab studies was derived from the score developed by Yao et al. ${ }^{16}$ The IFN score for each sample was calculated as the fold change between the median of the normalized expression of 21 probe sets in the sample and the average of the median values in the healthy volunteers. Then, patients were categorized as low or high IFN gene signature based on a bimodal distribution. ${ }^{15}$ This 21 -gene score was used to assess IFN gene signature in several trials including Phase I sifalimumab study, ${ }^{41}$ Phase II alpha-kinoid study ${ }^{42}$ and more recently in Phase II ustekinumab study. ${ }^{43}$ A 4-gene score (IFI27, IFI44, IFI44L and RSAD2) derived from the 21-gene score was next developed ${ }^{44}$ and used to assess IFN gene signature in sifalimumab and anifrolumab Phase II studies. ${ }^{22,45}$ In the anifrolumab Phase II study, the SRI-4 response proportions, in the high IFN gene signature population, were $52.0 \%$ for the anifrolumab $300 \mathrm{mg}$ group $(p<0.001), 38.5 \%$ for the $1 \mathrm{~g}$ group $(p=0.013)$ and $19.7 \%$ for placebo. Conversely, in the patients with a low IFN gene signature subgroup, no significant differences from placebo were seen. However, observed differences were rather due to an important difference in placebo effects between these two groups (high versus low IFN signature) than different response rates to anifrolumab in both subgroups. ${ }^{22}$ More recently, a post-hoc analysis compared anifrolumab $300 \mathrm{mg}$ with placebo on arthritis and rash measures using different outcomes. ${ }^{46}$ Skin involvement was assessed using two non-specific tools SLEDAI-2K and BILAG and one specific tool the Cutaneous Lupus Erythematosus Disease Area and Severity Index (CLASI). ${ }^{47} \mathrm{~A} \geq 50 \%$ decrease from baseline at week 52 of mCLASI (modified CLASI excluding oral ulcer and alopecia without scalp inflammation) was used to define cutaneous improvement. Using both mCLASI and nonspecific tools, a significant improvement of cutaneous involvement was observed in anifrolumab-treated patients compared with placebo in the high IFN gene signature subgroup. Conversely, there was no significant difference between anifrolumab and placebo for skin improvement using SLEDAI-2K and mCLASI in the low IFN gene signature subgroup. Moreover, improvement in arthritis was assessed using SLEDAI-2K and BILAG. Again, a significant improvement of arthritis was observed with anifrolumab $300 \mathrm{mg}$ compared with placebo in the high but not in the low IFN gene signature subgroup. ${ }^{46}$ These results suggested that patients with high IFN gene signature may represent a population more likely to benefit from the add-on anifrolumab therapy to SOC. It is conceivable that this stronger effect on patients with IFN could restrict the use of anifrolumab to these patients. Routine search for the presence of an IFN signature is not currently widely available. However, it is hoped that ultrasensitive IFN assay techniques will become routinely available in the near future. This would likely allow the selection of patients who may benefit from anifrolumab.

Anifrolumab is one of the more advanced drugs currently in the SLE pipeline. The release of the completed TULIP pivotal trials will inform us of its ability to confirm the hopes that were gleaned from its positive Phase IIb results and its positioning in the SLE armamentarium.

\section{Conclusion}

Targeting directly IFN receptors and blocking the action of all type I IFNs (IFN- $\alpha$, IFN- $\beta$ and IFN- $\omega$ ) appear to be of potential interest in SLE. Anifrolumab (previously MEDI546), a fully human monoclonal antibody that binds to subunit 1 of IFNAR1, showed promising preliminary results in its Phase IIb trial. The TULIP 1 Phase III trial did not meet its primary endpoint. The results of subsequent Phase II and Phase III trials are pending and will tell us more about the potential of anifrolumab as a new SLE treatment.

\section{Acknowledgments}

Laurent Arnaud has received honoraria or research funding (related to SLE) from Alexion, Amgen, Astra-Zeneca, GSK, Janssen, Lilly, Pfizer, Novartis, Roche, Springer healthcare.

Renaud Felten has received honoraria (not related to SLE) from AbbVie, BMS and Pfizer.

François Chasset has received honoraria (related to SLE) from GSK and Celgene.

Flora Sagez has received honoraria (not related to SLE) from BMS and Pfizer.

\section{Disclosure}

The authors report no conflicts of interest in this work. 


\section{References}

1. Felten R, Scher F, Sibilia J, Chasset F, Arnaud L. Advances in the treatment of systemic lupus erythematosus: from back to the future, to the future and beyond. Jt Bone Spine Rev Rhum. 2018. doi:10.1016/j.jbspin.2018.09.004

2. Felten R, Sagez F, Gavand P-E, et al. 10 most important contemporary challenges in the management of SLE. Lupus Sci Med. 2019;6(1): e000303. doi:10.1136/lupus-2018-000303

3. Felten R, Dervovic E, Chasset F, et al. The 2018 pipeline of targeted therapies under clinical development for systemic lupus erythematosus: a systematic review of trials. Autoimmun Rev. 2018;17 (8):781-790. doi:10.1016/j.autrev.2018.02.011

4. Chasset F, Arnaud L. Targeting interferons and their pathways in systemic lupus erythematosus. Autoimmun Rev. 2018;17(1):44-52. doi:10.1016/j.autrev.2017.11.009

5. Isaacs A, Lindenmann J. Virus interference. I. The interferon. Proc R Soc Lond B Biol Sci. 1957;147(927):258-267.

6. Lauwerys BR, Ducreux J, Houssiau FA. Type I interferon blockade in systemic lupus erythematosus: where do we stand? Rheumatol Oxf Engl. 2014;53(8):1369-1376. doi:10.1093/rheumatology/ ket403

7. Steinberg AD, Baron S, Talal N. The pathogenesis of autoimmunity in New Zealand mice, I. Induction of antinucleic acid antibodies by polyinosinic-polycytidylic acid. Proc Natl Acad Sci U S A. 1969;63 (4):1102-1107.

8. Hooks JJ, Moutsopoulos HM, Geis SA, Stahl NI, Decker JL, Notkins AL. Immune interferon in the circulation of patients with autoimmune disease. $N$ Engl J Med. 1979;301(1):5-8. doi:10.1056/ NEJM197907053010102

9. Rönnblom LE. Autoimmunity after alpha-interferon therapy for malignant carcinoid tumors. Ann Intern Med. 1991;115(3):178. doi:10.7326/0003-4819-115-3-178

10. Baechler EC, Batliwalla FM, Karypis G, et al. Interferon-inducible gene expression signature in peripheral blood cells of patients with severe lupus. Proc Natl Acad Sci. 2003;100(5):2610-2615. doi: 10.1073/pnas.0337679100

11. Bennett L, Palucka AK, Arce E, et al. Interferon and granulopoiesis signatures in systemic lupus erythematosus blood. J Exp Med. 2003;197(6):711-723. doi:10.1084/jem.20021553

12. Relle M, Weinmann-Menke J, Scorletti E, Cavagna L, Schwarting A. Genetics and novel aspects of therapies in systemic lupus erythematosus. Autoimmun Rev. 2015;14(11):1005-1018. doi:10.1016/j.autrev. 2015.07.003

13. Lausch E, Janecke A, Bros M, et al. Genetic deficiency of tartrate-resistant acid phosphatase associated with skeletal dysplasia, cerebral calcifications and autoimmunity. Nat Genet. 2011;43 (2):132-137. doi:10.1038/ng.749

14. Crow YJ. Type I interferonopathies: a novel set of inborn errors of immunity. Ann N Y Acad Sci. 2011;1238(1):91-98. doi:10.1111/ j.1749-6632.2011.06220.x

15. Hoffman RW, Merrill JT, Alarcón-Riquelme MME, et al. Gene expression and pharmacodynamic changes in 1,760 systemic lupus erythematosus patients from two phase III trials of BAFF blockade with tabalumab. Arthritis Rheumatol Hoboken NJ. 2017;69 (3):643-654. doi:10.1002/art.39950

16. Yao Y, Higgs BW, Morehouse C, et al. Development of potential pharmacodynamic and diagnostic markers for anti-IFN- $\alpha$ monoclonal antibody trials in systemic lupus erythematosus. Hum Genomics Proteomics. 2009;2009. doi:10.4061/2009/374312

17. Banchereau R, Hong S, Cantarel B, et al. Personalized immunomonitoring uncovers molecular networks that stratify lupus patients. Cell. 2016;165(3):551-565. doi:10.1016/j.cell. 2016.03.008
18. Nikpour M, Dempsey AA, Urowitz MB, Gladman DD, Barnes DA. Association of a gene expression profile from whole blood with disease activity in systemic lupus erythaematosus. Ann Rheum Dis. 2008;67(8):1069-1075. doi:10.1136/ard.2007.074765

19. Petri M, Singh S, Tesfasyone H, et al. Longitudinal expression of type I interferon responsive genes in systemic lupus erythematosus. Lupus. 2009;18(11):980-989. doi:10.1177/0961203309105529

20. Ivashkiv LB, Donlin LT. Regulation of type I interferon responses. Nat Rev Immunol. 2014;14(1):36-49. doi:10.1038/nri3581

21. Wallace DJ, Furie RA, Tanaka Y, et al. Baricitinib for systemic lupus erythematosus: a double-blind, randomised, placebo-controlled, phase 2 trial. Lancet Lond Engl. 2018;392(10143):222-231. doi:10.1016/S0140-6736(18)31363-1

22. Furie R, Khamashta M, Merrill JT, et al. Anifrolumab, an antiinterferon- $\alpha$ receptor monoclonal antibody, in moderate-to-severe systemic lupus erythematosus. Arthritis Rheumatol Hoboken NJ. 2017;69(2):376-386. doi:10.1002/art.39962

23. Uzé G, Lutfalla G, Gresser I. Genetic transfer of a functional human interferon alpha receptor into mouse cells: cloning and expression of its cDNA. Cell. 1990;60(2):225-234.

24. Novick D, Cohen B, Rubinstein M. The human interferon alpha/beta receptor: characterization and molecular cloning. Cell. 1994;77 (3):391-400.

25. Cohen B, Novick D, Barak S, Rubinstein M. Ligand-induced association of the type I interferon receptor components. Mol Cell Biol. 1995;15(8):4208-4214.

26. Oganesyan V, Gao C, Shirinian L, Wu H, Dall'Acqua WF. Structural characterization of a human $\mathrm{Fc}$ fragment engineered for lack of effector functions. Acta Crystallogr D Biol Crystallogr. 2008;64(Pt 6):700-704. doi:10.1107/S0907444908007877

27. Peng L, Oganesyan V, Wu H, Dall'Acqua WF, Damschroder MM. Molecular basis for antagonistic activity of anifrolumab, an antiinterferon- $\alpha$ receptor 1 antibody. mAbs. 2015;7(2):428-439. doi:10.1080/19420862.2015.1007810

28. Lu J, Chuntharapai A, Beck J, et al. Structure-function study of the extracellular domain of the human IFN-alpha receptor (hIFNAR1) using blocking monoclonal antibodies: the role of domains 1 and 2. J Immunol Baltim Md 1950. 1998;160(4):1782-1788.

29. Benoit P, Maguire D, Plavec I, Kocher H, Tovey M, Meyer F. A monoclonal antibody to recombinant human IFN-alpha receptor inhibits biologic activity of several species of human IFN-alpha, IFN-beta, and IFN-omega. Detection of heterogeneity of the cellular type I IFN receptor. J Immunol Baltim Md 1950. 1993;150(3):707-716.

30. Riggs JM, Hanna RN, Rajan B, et al. Characterisation of anifrolumab, a fully human anti-interferon receptor antagonist antibody for the treatment of systemic lupus erythematosus. Lupus Sci Med. 2018;5(1):e000261. doi:10.1136/lupus-2018-000261

31. Hall JC, Rosen A. Type I interferons: crucial participants in disease amplification in autoimmunity. Nat Rev Rheumatol. 2010;6(1):40-49. doi:10.1038/nrrheum.2009.237

32. Liao AP, Salajegheh M, Morehouse C, et al. Human plasmacytoid dendritic cell accumulation amplifies their type 1 interferon production. Clin Immunol Orlando Fla. 2010;136(1):130-138. doi:10.1016/j.clim.2010.02.014

33. Brohawn P, Santiago L, Morehouse C, Higgs B, Illei G, Ranade K. Target modulation of a type I interferon gene signature and pharmacokinetics of anifrolumab in a phase IIb study of patients with moderate to severe systemic lupus erythematosus. ACR Meet Abstr. 2015. Available from: https://acrabstracts.org/ abstract/target-modulation-of-a-type-i-interferon-gene-signatureand-pharmacokinetics-of-anifrolumab-in-a-phase-iib-study-ofpatients-with-moderate-to-severe-systemic-lupus-erythematosus/. Accessed January 10, 2019. 
34. Tummala R, Rouse T, Berglind A, Santiago L. Safety, tolerability and pharmacokinetics of subcutaneous and intravenous anifrolumab in healthy volunteers. Lupus Sci Med. 2018;5(1):e000252. doi:10.1136/ lupus-2017-000252

35. Tanaka Y, Takeuchi T, Okada M, et al. Safety and tolerability of anifrolumab, a monoclonal antibody targeting type I interferon receptor, in Japanese patients with systemic lupus erythematosus: a multicenter, phase 2, open-label study. Mod Rheumatol. 2019; (ja):1-18. doi:10.1080/14397595.2019.1583833

36. Update on TULIP 1 phase III trial for anifrolumab in systemic lupus erythematosus. Available from: https://www.astrazeneca. com/media-centre/press-releases/2018/update-on-tulip-1-phase-iiitrial-for-anifrolumab-in-systemic-lupus-erythematosus-31082018. html. Accessed January 10, 2019.

37. Mahieu MA, Strand V, Simon LS, Lipsky PE, Ramsey-Goldman R. A critical review of clinical trials in systemic lupus erythematosus. Lupus. 2016;25(10):1122-1140. doi:10.1177/0961203316652492

38. Navarra SV, Guzmán RM, Gallacher AE, et al. Efficacy and safety of belimumab in patients with active systemic lupus erythematosus: a randomised, placebo-controlled, phase 3 trial. Lancet. 2011;377 (9767):721-731. doi:10.1016/S0140-6736(10)61354-2

39. Furie R, Petri M, Zamani O, et al. A phase III, randomized, placebo-controlled study of belimumab, a monoclonal antibody that inhibits B lymphocyte stimulator, in patients with systemic lupus erythematosus. Arthritis Rheum. 2011;63(12):3918-3930. doi:10.1002/ art.30613

40. Touma Z, Gladman DD. Current and future therapies for SLE: obstacles and recommendations for the development of novel treatments. Lupus Sci Med. 2017;4(1):e000239. doi:10.1136/lupus-2017-000239

41. Merrill JT, Wallace DJ, Petri M, et al. Safety profile and clinical activity of sifalimumab, a fully human anti-interferon $\alpha$ monoclonal antibody, in systemic lupus erythematosus: a phase I, multicentre, double-blind randomised study. Ann Rheum Dis. 2011;70 (11):1905-1913. doi:10.1136/ard.2010.144485
42. Ducreux J, Houssiau FA, Vandepapelière P, et al. Interferon $\alpha$ kinoid induces neutralizing anti-interferon $\alpha$ antibodies that decrease the expression of interferon-induced and B cell activation associated transcripts: analysis of extended follow-up data from the interferon $\alpha$ kinoid phase I/II study. Rheumatol Oxf Engl. 2016;55(10):1901-1905. doi:10.1093/rheumatology/ kew262

43. van Vollenhoven RF, Hahn BH, Tsokos GC, et al. Efficacy and safety of ustekinumab, an IL-12 and IL-23 inhibitor, in patients with active systemic lupus erythematosus: results of a multicentre, double-blind, phase 2, randomised, controlled study. Lancet Lond Engl. 2018;392(10155):1330-1339. doi:10.1016/S0140-6736(18) 32167-6

44. Yao Y, Higgs BW, Richman L, White B, Jallal B. Use of type I interferon-inducible mRNAs as pharmacodynamic markers and potential diagnostic markers in trials with sifalimumab, an antiIFN $\alpha$ antibody, in systemic lupus erythematosus. Arthritis Res Ther. 2010;12(Suppl 1):S6. doi:10.1186/ar2887

45. Khamashta M, Merrill JT, Werth VP, et al. Sifalimumab, an antiinterferon- $\alpha$ monoclonal antibody, in moderate to severe systemic lupus erythematosus: a randomised, double-blind, placebocontrolled study. Ann Rheum Dis. 2016;75(11):1909-1916. doi:10.1136/annrheumdis-2015-208562

46. Merrill JT, Furie R, Werth VP, et al. Anifrolumab effects on rash and arthritis: impact of the type I interferon gene signature in the phase IIb MUSE study in patients with systemic lupus erythematosus. Lupus Sci Med. 2018;5(1):e000284. doi:10.1136/ lupus-2018-000284

47. Albrecht J, Taylor L, Berlin JA, et al. The CLASI (Cutaneous lupus erythematosus disease area and severity index): an outcome instrument for cutaneous lupus erythematosus. $J$ Invest Dermatol. 2005;125(5):889-894. doi:10.1111/j.0022202X.2005.23889.x
Drug Design, Development and Therapy

\section{Publish your work in this journal}

Drug Design, Development and Therapy is an international, peerreviewed open-access journal that spans the spectrum of drug design and development through to clinical applications. Clinical outcomes, patient safety, and programs for the development and effective, safe, and sustained use of medicines are a feature of the journal, which has also

\section{Dovepress}

been accepted for indexing on PubMed Central. The manuscript management system is completely online and includes a very quick and fair peer-review system, which is all easy to use. Visit http://www. dovepress.com/testimonials.php to read real quotes from published authors. 